\title{
Streptococcus sanguinis
}

National Cancer Institute

\section{Source}

National Cancer Institute. Streptococcus sanguinis. NCI Thesaurus. Code C86806.

A species of facultatively anaerobic, Gram positive, cocci shaped bacteria in the phylum Firmicutes. This species is alpha hemolytic, arginine deaminase positive, hydrolysis esculin, and urease and catalase negative. It can ferment inulin and trehalose but not mannitol, sorbitol, or raffinose. S. sanguinis is found in the normal oral flora of humans and is a pathogen that can cause dental caries, and endocarditis after dental injury. 